ÉGYPTE monde arabe

\section{Égypte/Monde arabe}

3 | 2006

Terrains d'Égypte, anthropologies contemporaines

\title{
Diaspora africaine, esclavage et Islam
}

À propos de Slavery on the Frontiers of Islam, Lovejoy Paul E. (dir.)

\section{Matthieu Fintz}

\section{(2) OpenEdition}

\section{Journals}

Édition électronique

URL : https://journals.openedition.org/ema/1725

DOI : $10.4000 /$ ema. 1725

ISSN : 2090-7273

Éditeur

CEDEJ - Centre d'études et de documentation économiques juridiques et sociales

Édition imprimée

Date de publication : 31 décembre 2006

Pagination : 203-213

ISSN : 1110-5097

Référence électronique

Matthieu Fintz, «Diaspora africaine, esclavage et Islam », Égypte/Monde arabe [En ligne], 3 | 2006, mis en ligne le 08 juillet 2008, consulté le 07 juillet 2022. URL : http://journals.openedition.org/ema/1725 ; DOI : https://doi.org/10.4000/ema.1725 


\title{
Matthieu Fintz
}

\section{DIASPORA AFRICAINE, ESCLAVAGE ET ISLAM}

\author{
À PROPOS DE SLAVERY ON THE FRONTIERS OF ISLAM, LOVEJOY
} PAUL E. (DIR.), PRINCETON, MARKUS WIENER PUBLISHERS, 2004, 297 P.

\begin{abstract}
C'est tout l'intérêt de Slavery on the Frontiers of Islam, ouvrage dirigé par Paul E. Lovejoy, que de revenir sur la formation des frontières entre Dâr al-Islâm et Dâr al-Harb, entre musulmans et non musulmans, entre hommes libres et esclaves et sur la place d'entre-deux du Bilâd al-Sûdân (le "Pays des Noirs ») dans cet écheveau de frontières. Ce faisant, les différents contributeurs confrontent aussi la logique scolastique de l'esclavage, telle que déployée dans les traités juridiques ou religieux, aux pratiques d'asservissement qui mettent en jeu les déterminations religieuses aussi bien que les configurations variées de différenciation sociale et politique. L'ouvrage est le fruit du travail accompli au sein du Nigerian Hinterland Project, programme de recherche hébergé par la York University (Toronto, Canada), et mené en relation avec I'UNESCO et des universités ouest africaines. L'objectif de ce programme est de documenter la constitution de la diaspora africaine issue de l'arrière-pays nigérian au temps de l'esclavage, de 1650 à 1900.

Dans cet ouvrage, Paul E. Lovejoy défend une " approche afro-centrique » dont le contenu n'est discuté que dans le dernier chapitre, même si le lecteur la devine dès les premières pages. Celle-ci consiste à " regarder vers l'extérieur depuis I'Afrique » (" to look outward from Africa ») (p. 234) et vise à contrebalancer la vision euro-centrique qui mettait davantage en valeur la subordination des esclaves à leur régime d'asservissement, au détriment d'une prise en compte des dynamiques, conflictuelles ou non, de production des identités au sein des populations asservies et des arrangements possibles entre
\end{abstract}

1. Cette interprétation "révisionniste" de l'histoire de l'esclavage est davantage explicitée dans Lovejoy P. E., 1997, «The African diaspora : revisionist interpretations 
diaspora et « homeland ${ }^{1}$. Cette perspective, qui appréhende les relations entre Islam et esclavage depuis les pratiques et points de vue de ceux qui ont constitué la diaspora africaine, explique en grande partie la volonté des auteurs de soumettre l'esclavage des mondes sub-saharien et atlantique au même questionnement en rapportant le regroupement de ces ensembles géographiques, culturels et politiques aux flux des populations asservies plutôt qu'aux idéologies de l'esclavage en Europe et dans le monde musulman. L'organisation de l'ouvrage suit ainsi les itinéraires d'esclavage depuis l'Afrique sub-saharienne, qui a retenu en son sein une partie importante des esclaves, jusqu'aux deux points d'arrivée de la diaspora africaine en Afrique du Nord et Méditerranée et aux Amériques.

La teneur des contributions réunies dans ce livre permet, par ailleurs, d'entrevoir rétrospectivement le chemin parcouru par les recherches menées sur l'esclavage et l'influence que les postcolonial, subaltern, et diaspora studies ont eu sur les questionnements pratiqués. Le champ d'étude se déploie désormais jusque sur les terrains de l'histoire orale ${ }^{2}$, permettant d'obtenir de précieux éléments biographiques sur les constructions identitaires à l'œuvre chez les esclaves eux-mêmes et leurs descendants. L'utilisation de la masse documentaire que constituent les manuscrits arabes d'Afrique de l'Ouest restés longtemps inexplorés est toujours une ressource importante des recherches publiées dans ce livre. De cette utilisation, il apparaît clairement que l'idée prévalente depuis les colonisations et jouant sur le couple absence d'écriture/ absence d'histoire du continent africain ne fut qu'un mythe arrangeant bien la « mission civilisatrice » des puissances européennes. Grâce à ces manuscrits, il est désormais possible de documenter I'histoire de l'Afrique indépendante. Et le livre de Paul E. Lovejoy le fait en adoptant de nouveaux questionnements et de nouvelles méthodologies. L'ouvrage propose en effet une stratégie de recherche qui s'éloigne des canons de l'histoire des idées islamiques relatives à l'esclavage pour se rapprocher d'une histoire des pratiques d'agency, c'està-dire des modalités historiques, donc flexibles et créatives, de perception de soi parmi les membres de la diaspora africaine.

Avant de revenir sur la présentation des études de cas de l'ouvrage dans lesquelles ces questionnements et méthodologies sont déployés, il est peut être utile de donner quelques caractéristiques de l'esclavage en terre d'Islam, tel qu'il a pu être codifié et exercé aux frontières méridionales de Dâr al-Islâm.

of ethnicity, culture and religion under slavery ", Studies in the World History of Slavery, Abolition and Emancipation, 2(1). L'ouvrage présenté ici élargit la problématique au commerce trans-saharien.

2. Stilwell S., Hamza I., Lovejoy P. E., Dako S., 2001, "The oral history of royal slavery in the Sokoto Caliphate : an interview with Sallama Dako », History in Africa, 28, p. 273-91. 


\section{ESCLAVAGE EN TERRE D'ISLAM ET MUSULMANS EN TERRE D'ESCLAVAGE}

"L'imam [i.e. le leader musulman dirigeant le jihâd] devrait prendre en considération le sort des prisonniers adultes mâles, et prendre celle d'entre les options suivantes qu'il considère la plus salutaire : les mettre à mort, les libérer sans pénalité, demander une rançon pour eux, demander la taxe de capitation (jizya), ou les mettre en esclavage ». Ainsi statue Khalîl ibn Ishâq al-Jundî, auteur d'une compilation juridique Mâlikî égyptienne du $14^{\mathrm{e}}$ siècle faisant autorité, sur les règles du jihâd ${ }^{3}$. La mise en esclavage de prisonniers n'est ainsi qu'une des cinq options. En même temps, le jihâd est la seule voie légitime possible autorisant l'asservissement selon les préceptes islamiques. Car, comme le rappelle encore l'érudit de Tombouctou Ahmad Bâbâ à la fin du $16^{\mathrm{e}}$ siècle: "La cause de l'esclavage est la non croyance. Tout esclave possédé doit avoir été capturé, ou un de ses ancêtres doit avoir été capturé » ${ }^{4}$. La mise en esclavage n'est donc possible, d'après la jurisprudence islamique (fiqh), qu'au-delà de Dâr al-Islâm.

Cependant, comme la parution même du traité d'Ahmad Bâbâ I'atteste, entre Dâr al-Islâm et Dâr al-Harb, « la porte du doute est restée ouverte pour ceux qui cherchaient un prétexte pour la possession $\aleph^{5} d^{\prime}$ esclaves. Le traité d'Ahmad Bâbâ constitue en effet une réponse à une requête des habitants de I'oasis saharien de Tuwât concernant les catégories de personnes légitimement réductibles à l'esclavage et s'étonnant de l'asservissement de populations du Bilâd al-Sûdân ayant embrassé I'Islam de longue date. La parution du traité indique ainsi qu'en dépit du critère religieux restrictif sur l'approvisionnement en esclaves, celui-ci était souvent ignoré au profit d'autres facteurs comme l'ethnicité, l'ascendance ou la couleur de peau utilisée pour discriminer qui était libre et qui pouvait être asservi ${ }^{6}$. La " porte du doute » demeurait d'autant plus entrouverte que les frontières de Dâr al-Islâm au Bilâd al-Sûdân étaient elles-mêmes fluctuantes selon les conjonctures historiques.

Il y a donc eu des musulmans asservis par d'autres musulmans en violation de la jurisprudence. La question de la mise en esclavage de musulmans noirs

3. Cité dans Hunwick J. O., Troutt Powell E., 2002, The African Diaspora in the Mediterranean Lands of Islam, Princeton, Markus Wiener publishers, p. 23.

4. Barbour B., Jacobs M., 1985, "The Mi'raj : a legal treatise on slavery by Ahmad Baba » dans Willis J. R. (dir.), Slaves and Slavery in Muslim Africa. 1- Islam and the ideology of enslavement, Londres, Franck Cass, p. 136.

5. Willis J. R., 1985, "The ideology of enslavement in Islam » dans Willis J. R. (dir.), op. cit., p. 6.

6. Sikainga A. A., 2000, « Comrades in arms or captives in bondage : sudanese slaves in the turco-egyptian army, 1821-1865 » dans Toru M., Philips J. E. (dirs), Slaves elites in the Middle East and Africa. A comparative study, Londres/New York, Kegan Paul International, p. 199-200. 
et, par là, la reconnaissance, implicite ou explicite, de critères ethniques ou raciaux à l'esclavage n'a cessé d'être posée sous l'effet des vagues d'islamisation dans le Bilâd al-Sûdân. Malgré leurs essais cartographiques de définition des terres d'Islam et des zones où la mise en esclavage pouvait s'exercer, les érudits n'ont souvent pas tranché radicalement la question, laissant une large place aux controverses ${ }^{7}$ dont l'issue accréditait parfois l'idée que le critère décisif pouvait, dans certains contextes, reposer sur la couleur de la peau ${ }^{8}$.

Le contexte du jihâd qui domine le Soudan occidental et central au $19^{\mathrm{e}}$ siècle ${ }^{9}$ est également marqué par l'asservissement de musulmans par d'autres musulmans. Ces esclaves pouvaient être des réformistes musulmans capturés au cours du jihâd sans être rachetés ou libérés ou des individus perçus comme musulmans "syncrétiques », corrompus ou ennemis du jihâd. Hors de ce contexte, il pouvait encore s'agir de commerçants musulmans attaqués et capturés le long des routes trans-sahariennes par des groupes pratiquant le banditisme et l'esclavagisme.

Le commerce trans-saharien fut non seulement un producteur d'esclaves, mais aussi un producteur d'esclaves musulmans. Comme le remarque John O. Hunwick dans la contribution que nous présenterons plus loin, des motifs économiques permettent aussi de rendre compte de l'écart entre la jurisprudence et les pratiques effectives d'asservissement. Sur les marchés d'esclaves de la Méditerranée, les esclaves noirs étaient en effet vendus en tant que musulmans, les marchands d'esclaves opérant la transformation du païen en musulman au cours du trajet trans-saharien. Circoncision, apprentissage des

7. Hunwick J. O., 1999, "Islamic law and polemics over slavery in North and West Africa, 16th-19th century », dans Marmon S. (dir.), Slavery in the Islamic Middle East, Princeton, Markus Wiener Publishers, p. 43-68; Hunwick J. O., 2000, " Ahmad Bâbâ on Slavery ", Sudanic Africa, 11, p. 131-139; Hunwick J. O., Troutt Powell E., 2002, The African Diaspora in the Mediterranean Lands of Islam, op. cit., p. 35-50.

8. Cela est d'autant plus vrai au fur et à mesure que l'on s'élève dans les hiérarchies entre esclaves. Nasser Rabbat souligne en effet que lors de son institutionnalisation le mot mamlûk ne fut jamais utilisé pour désigner les esclaves noirs, même lorsque ceux-ci servaient exclusivement comme guerriers, comme ils le firent durant la période fatimide en Égypte. Ainsi, le mot mamlûk en vint à avoir des connotations géographiques et ethniques : «il signifiait des jeunes hommes blancs, principalement des turcs ou turquisés», en l'occurrence des Circassiens, des Turcs ou des Mongols, Rabbat N., "The Changing Concept of Mamlûk in the Mamluk Sultanate in Egypt and Syria » dans Toru M., Philips J. E. (dirs), Slaves Elites in the Middle East and Africa. A comparative study, op. cit., p. 82.

9. Sur le contexte intellectuel de I'Islam au Soudan occidental à cette période, Willis J. R., 1967, "Jihad fi Sabil Allah - Its doctrinal basis in Islam and some aspects of its evolution in nineteenth-century West Africa », Journal of African History, 8(3), p. 395-415. 
rudiments de la prière en arabe, ou encore imposition d'un nom arabe étaient supposés offrir un argument commercial et un gage de probité de la marchandise à une clientèle réservée sur l'incursion de non musulmans dans le foyer et l'intimité familiale ${ }^{10}$. Devenus musulmans, les esclaves ont dès lors multiplié les façons d'être musulman.

\section{LES FRONTIÈRES DE L'ISLAM}

Le chapitre introductif de Paul Lovejoy permet de donner toute sa dimension à la notion de frontières telle qu'elle est appliquée à l'examen des relations multiples entre esclavage et Islam. Les frontières de I'Islam sont représentées par I'assemblage de plusieurs couches. Trois modalités de la frontière structurent en fait l'ensemble des contributions. Il s'agit d'abord des frontières géographiques de l'Islam dont les contours sont dessinés dans les stratégies d'expansion des États soudaniens aux dépens des populations voisines. Ces frontières sont les sources de constitution de la diaspora africaine qui est ensuite dirigée vers les ports du commerce transatlantique ou à travers le Sahara vers les villes de la Méditerranée, ou encore abandonnée aux marchés d'esclaves des États et royaumes soudaniens. À la lisière des émirats musulmans qui se constituent au début du $19^{\mathrm{e}}$ siècle, ces frontières mobiles de l'esclavage sont aussi le lieu de discrimination entre Dâr al-Islâm et Dâr al-Harb. La référence géographique des frontières de I'Islam en Afrique soudanienne est ainsi redoublée par la frontière symbolique entre musulmans et non musulmans. En effet, I'esclavage a interrogé la définition même de ce qu'est un musulman. L'intégration croissante de populations noires du Bilâd al-Sûdân au monde musulman a d'abord conduit au questionnement des critères de l' « esclavage juste » mis à l'épreuve par les pratiques d'asservissement de populations noires musulmanes. Les érudits soudanais, interrogés par les souverains musulmans de ces zones frontalières, ont tenté de

10. Une des caractéristiques de l'esclavage dans l'Empire ottoman, par rapport à l'esclavage transatlantique, réside en effet dans I'utilisation massive d'esclaves à des fins domestiques. Cette caractéristique a été mobilisée par les avocats de l'esclavage pour contrer les campagnes abolitionnistes britanniques dans la première moitié du $19^{\mathrm{e}}$ siècle en défendant l'idée d'un esclavage doux se déployant dans l'ambiance feutrée des harems royaux et passant sous silence à la fois l'esclavage agricole, la différenciation sociale entre formes d'esclavage domestique et la différenciation raciale calculée sur la couleur de peau (pour les concubines notamment). Voir à ce sujet, Baer G., 1967, "Slavery in Nineteenth Century Egypt », Journal of African History, 8(3), p. 417-41; Toledano E. R., 1998, Slavery and Abolition in the Ottoman Middle East, Seattle/ London, University of Washington Press; Troutt Powell E. M., 2003, A Different Shade of Colonialism. Egypt, Great Britain and the mastery of Sudan, Berkeley - Los Angeles - Londres, University of California Press. 
répondre à cette question. À un second niveau, par leurs pratiques religieuses déployées dans leur société d'accueil (musulmanes ou non), les esclaves musulmans d'Afrique soudanienne ont aussi réinventé ce qu'être musulman veut dire et, par là, ont redéfini les frontières de la pratique religieuse islamique. Les frontières de I'Islam sont enfin entendues dans cet ouvrage comme les lieux atteints par la diaspora africaine musulmane, tant en Afrique du Nord et au MoyenOrient que dans les Amériques, démontrant ainsi la contribution du mouvement des Africains asservis à la formation du monde musulman moderne.

\section{LES ZONES FRONTIÈRES DE L'ESCLAVAGE DANS LE BILÂD AL-SÛDÂN}

Les six chapitres suivants rendent compte de l'organisation du commerce d'esclaves et de la différenciation sociale du monde des esclaves sur les lieuxfrontières de constitution de la diaspora africaine aux frontières soudaniennes du monde de I'Islam au $18^{\mathrm{e}}$ et, surtout, au $19^{\mathrm{e}}$ siècles. Les sites explorés dans les contributions correspondent à l'espace de développement du califat de Sokoto ${ }^{11}$ à partir du début du $19^{\mathrm{e}}$ siècle et au Soudan central dans le contexte du jihâd inauguré par Usman dan Fodio en 1804-9 et poursuivi par ses successeurs tout au long du siècle.

La contribution de George Michael La Rue est particulièrement éclairante sur la constitution d'une zone frontière d'asservissement au Bagirmi (royaume occidental du Tchad contemporain) et son insertion dans le réseau des routes commerciales et de pèlerinage traversant les émirats du Soudan central et le Sahara pour atteindre les villes méditerranéennes de Tripoli et de Benghazi, les ports de la mer Rouge en Égypte ou encore les villes d'Assiout et de Minia le long de la vallée du Nil. Il montre comment le tracé de ces routes et I'utilisation du Bagirmi comme réservoir d'esclaves dépendaient des politiques des gouvernements situés le long et à la fin de ces routes. Le rôle de la politique

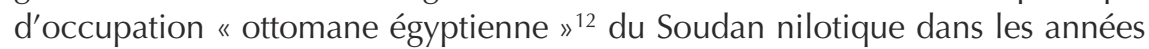

11. Les frontières atteintes par le califat de Sokoto durant cette période s'étendent depuis la zone sahélienne du Burkina Faso actuel à l'Ouest, pour englober le Nord Nigeria jusqu'au Nord Cameroun actuel à l'Est.

12. Nous reprenons ici I'appellation utilisée par l'auteur et par ses références à Toledano E. R., 1998, Slavery and Abolition in the Ottoman Middle East, Seattle/ London, University of Washington Press; Toledano E. R., 2003, State and Society in mid-nineteenth-century Egypt, Cambridge, Cambridge University Press; Troutt Powell E. M., 2003, A Different Shade of Colonialism. Egypt, Great Britain and the mastery of Sudan, Berkeley/Los Angeles/Londres, University of California Press. Ces différents travaux parlent de l'émergence d'une élite ottomane-égyptienne au moment de l'arrivée au pouvoir de Muhammad Ali. En porte-à-faux avec les récits de l'histoire nationaliste officielle en Égypte, ces études situent Muhammad Ali comme un simple gouverneur 
1820 dans le transfert d'esclaves du Bagirmi à la vallée du Nil est par exemple évoqué. Dans ce contexte, I'occupation du Soudan nilotique, cumulée aux pressions britanniques sur l'Empire ottoman pour abolir l'esclavage, est un des éléments déclencheurs de l'émergence de la Zarîba. Le recrutement des esclaves, engageant également le commerce d'ivoire, n'est alors plus directement mené par des États mais par des slave raiders privés du Nord Soudan qui fuyaient la sécheresse de 1834-1837, les taxations du régime « turc » et la réforme agraire ayant abouti à la constitution de vastes domaines privés reposant sur le travail des esclaves ${ }^{13}$. La sécheresse de cette décennie le long de la vallée du Nil impose également un sérieux coup de frein à la demande d'esclaves par l'agriculture égyptienne. Ces perturbations politiques et écologiques de la voie commerciale Bagirmi - Darfour - Assiout conduisent à son délaissement par les caravanes au profit d'une nouvelle route reliant le Bagirmi à Wadai (Tchad central contemporain) puis à Benghazi, le commerce d'esclaves sur la route d'Assiout ne reprenant qu'après la décennie 1840 et perdurant audelà de l'abolition décidée par l'Empire ottoman en 1857 en raison de la forte demande de main-d'œuvre induite par le boom cotonnier égyptien.

Une place importante dans ces six chapitres est accordée à l'étude du groupe des " esclaves royaux » formant une part grandissante de l'élite militaire et administrative des émirs au fur et à mesure de la routinisation du jihâd. La contribution de Sean Stilwell est particulièrement stimulante à deux points de vue. Il étend la notion de mamlûk au-delà de ses contextes habituels de formation et de transformation au Moyen-Orient pour la faire fonctionner dans le contexte du califat de Sokoto. II montre comment ce groupe, fortement différencié, d'esclaves royaux est devenu un creuset pour la constitution et l'acquisition de savoirs de gouvernement, à la fois militaires et administratifs ${ }^{14}$. Mais le développement de ce type d'esclavage concomitant de la prise de pouvoir par les émirs de Sokoto n'est pas tant rapporté par lui à la forte inspiration islamique de ces mouvements politiques qu'au processus de centralisation du pouvoir à

ottoman, plutôt que comme un leader nationaliste égyptien visant à libérer l'Égypte du joug ottoman. On peut rappeler aussi que, dans I'histoire du Soudan nilotique, la période d'occupation par les armées de Muhammad Ali est nommée la Turkiyya, indiquant que le point de vue afro-centrique légitimerait la nature « ottomane-égyptienne » du gouvernement de Muhammad Ali.

13. Sur ce point, Spaulding J., 1982, "Slavery, land tenure and social class in the Northern Turkish Sudan », International Journal of African historical Studies, 15(1), p. 1-20.

14. Dans une publication antérieure, Sean Stilwell suggère, en référence à Pierre Bourdieu, de penser l'acquisition d'esclaves royaux comme une accumulation de " capital culturel », Stilwell S., 2000, "The power of knowledge and the knowledge of power: kinship, community and royal slavery in pre-colonial Kano, 1807-1903 » dans Toru M., Philips J. E. (dirs), Slave Elites in the Middle East and Africa, Londres/ New York, Kegan Paul International, p. 117-56. 
I'intérieur du califat. Affrontant I'hostilité de I'aristocratie hausa et le risque de factionnalisme, les leaders du jihâd ont trouvé dans le recrutement d'esclaves royaux le moyen de se maintenir en disposant d'une garde rapprochée tendant à devenir un intermédiaire obligé dans tout un ensemble de transactions (collecte d'impôts, gestion de la terre, protocole) : "Les esclaves étaient utilisés parce qu'il y avait un impératif politique à agir ainsi » (p. 103). Cette étude stimulante permet ainsi de répondre en partie à la question souvent explorée du rôle de I'Islam dans I'accroissement du commerce d'esclaves en Afrique sub-saharienne au $19^{\text {e }}$ siècle, en rapportant cette concomitance aux dynamiques politiques de l'exercice du pouvoir dans ces régions plutôt qu'à des principes religieux islamiques qui seraient foncièrement esclavagistes. L'Islam ne fut qu'une, quoique importante, variable de la mise en esclavage et de l'accroissement de l'esclavage dans cette région au cours du $19^{\mathrm{e}}$ siècle.

La différenciation du monde des esclaves fait encore l'objet de la contribution de John Edward Philips sur les Ribât, campements fortifiés et zones frontières par excellence, dont la présence est attestée avant le $19^{\text {e }}$ siècle, mais qui sont développés au cours de l'installation du califat de Sokoto. L'auteur montre que dans ses fonctions multiples de défense des routes commerciales et des frontières du califat, de centres de production d'artisanat, de moyen de sédentariser les pasteurs fulani, de mêler I'Islam fulani et hausa à l'intérieur d'une conception jihadiste et, enfin, d'entrepôt d'esclaves, les Ribât pourraient bien avoir signifié la naissance d'une culture islamique hybride au sein de laquelle les esclaves auraient joué un rôle important. Le Ribât est en fait essentiel au développement d'une classe d'esclaves fonctionnaires, les mamlûk du califat, qui vont acquérir une place importante dans la structure politique mais aussi dans le recrutement d'esclaves et leur gestion. Ces esclaves royaux pouvaient détenir eux-mêmes des esclaves et le système des plantations royales, grandes utilisatrices d'esclaves, pouvait également être entre leurs mains.

Seule la contribution de Ibrahim Hamza quitte les sphères de l'élite des esclaves pour revenir sur la situation des esclaves non royaux du califat, désignés par le terme de Gwarawa insistant sur le fait qu'ils parlent une langue différente du Hausa et qu'ils sont considérés comme d'origine non musulmane. Ils forment sans doute la plus grande partie de la population d'esclaves de l'émirat de Kano qui, lors de son incorporation au califat de Sokoto en 1806, représente la moitié de la population totale de l'émirat. L'auteur montre comment ce commerce s'est déroulé dans le cadre du développement de l'agriculture à grande échelle sur les plantations destinées à alimenter le palace de l'émir.

\section{Esclaves noirs et frontières de la pratique religieuse islamique au Maghreb}

Deux contributions concourent à documenter les processus d'imposition d'identités et de redéfinition des frontières de la pratique religieuse islamique induits par la présence d'esclaves musulmans noirs au Maghreb. Elles 
traitent du culte Bori en Tunisie aux $18^{\mathrm{e}}$ et $19^{\mathrm{e}}$ siècles. À l'instar du Zâr en Éthiopie, au Soudan, en Égypte ou dans la péninsule arabique, le Bori est un culte de possession développé au Maghreb par les esclaves importés du Bilâd al-Sûdân (principalement des esclaves hausa). D'après la contribution de John O. Hunwick, on peut penser que les lieux d'exercice du culte Bori ont pu servir de zones de contact entre des pratiques stigmatisées comme païennes et des pratiques musulmanes. II montre en effet que les esclaves sub-sahariens ont trouvé dans les sociétés maghrébines une structure d'opportunités caractérisée par un nombre potentiellement infini de jinn et au sein de laquelle des systèmes de croyance sub-sahariens ont pu s'intégrer et permettre à leurs porteurs de faire face au traumatisme psychologique de l'asservissement. "Des croyances et des pratiques prenant leur source en Afrique sub-saharienne ont été accommodées à l'intérieur d'une structure islamique généreuse et sont devenues une influence importante dans la vie religieuse des Nord Africains » (p. 167).

Dans sa contribution, Ismael Musah Montana souligne également que la prégnance du soufisme dans la vie religieuse tunisienne aux $18^{\mathrm{e}}$ et $19^{\mathrm{e}}$ siècles et le pluralisme religieux (les cultes de saints en particulier) ont certainement concouru à faire entrer le culte Bori dans l'espace religieux légitime tunisien. Mais, par l'examen des écrits de l'érudit Ahmad ibn al-Qâdî al-Timbuktâwî, il insiste sur le fait que la frontière entre pratiques religieuses légitimes et illégitimes fut âprement discutée et controversée au tournant du $19^{\text {e }}$ siècle, conjoncture marquée à la fois par le jihâd en Afrique de l'Ouest et le mouvement wahhâbî en Arabie, ce dernier s'érigeant contre les innovations (bid'a) de I'Islam populaire comme les cultes de saints ou le soufisme. Fils d'un qâdî de Tombouctou, éduqué dans la ville sainte de Jenne avant de revenir à Tombouctou, al-Timbuktâwî écrit en 1808-9 deux opuscules dans lequel il dénonce la pratique du culte Bori telle qu'il a pu l'observer en Tunisie à son retour de pèlerinage. L'attaque d'al-Timbuktâwî cible non seulement les adeptes du culte qu'il accuse d'hérésie (shirk) et d'incroyance (kufr) mais plus encore les praticiens du culte pour lesquels il réclame l'interdiction de pratiquer, la persécution et le maintien en esclavage comme juste punition à leur déviance religieuse. Les femmes prêtresses du culte Bori sont particulièrement frappées par le courroux de l'érudit qui les représente comme des sources de dévoiement et de lesbianisme (musâhaqa). Selon l'auteur, les vues dogmatiques et puritaines d'al-Timbuktâwî reconnaissent explicitement une relation entre identité ethnique et légalité de l'esclavage. Pour preuve, son utilisation de la catégorie des Sûdân Tûnis, terme qui discrimine à l'intérieur de la communauté des wusfân (domestiques) la portion des esclaves nés dans leurs patries d'origine et arrivés récemment en Tunisie et qui, selon al-Timbuktâwî, sont les principaux propagateurs du culte. La condamnation du culte Bori s'intègre ainsi au projet plus vaste de définition des critères de l'esclavage, voire de ségrégation sociale (il promeut par exemple une législation interdisant le mariage entre wusfân et femmes musulmanes). 


\section{Esclaves musulmans aux Amériques}

Les trois derniers chapitres, tous co-signés par Paul E. Lovejoy, sont des tentatives exemplaires pour retracer les pratiques d'agency déployées par les esclaves africains musulmans dans les Amériques, figurant ainsi une nouvelle frontière de I'Islam. Ces contributions s'inscrivent dans la discussion sur l'importance des processus de créolisation, de résistance et/ou d'ajustements (accommodation) des rapports de la diaspora africaine à ses sociétés d'accueil. Pour les auteurs, la diaspora d'africains musulmans asservis met en question les processus de créolisation qui décrivent un background africain totalement reforgé dans la culture américaine. Ils partent de l'hypothèse inverse, d'une continuité entre ce que les esclaves musulmans africains pouvaient espérer aux Amériques compte tenu de leurs caractéristiques sociales et de la fréquentation antérieure de l'esclavage dans leurs sociétés musulmanes d'origine en Afrique de l'Ouest.

Le profil sociographique des africains musulmans asservis et déportés aux Amériques en fait une population, certes différenciée, mais qui regroupe un certain nombre de caractéristiques communes. D'abord, ils sont relativement peu nombreux. Or, malgré cette faiblesse numérique, il apparaît que le nombre de manumission est relativement plus important pour les esclaves musulmans que pour les autres ${ }^{15}$. Ensuite, il s'agit d'une population essentiellement mâle, jeune et urbaine ${ }^{16}$. Enfin, ces esclaves africains musulmans proviennent souvent de familles de commerçants ou des élites aristocratiques et militaires des États musulmans d'Afrique de I'Ouest qui possèdent elles-mêmes des esclaves. De ce fait, ils possèdent une éducation religieuse, un savoir pratique et une connaissance des textes arabes définissant les conditions de la mise en esclavage légitime. Ces esclaves musulmans avaient dès lors des « raisons légitimes de rechercher leur liberté au titre que, en tant que nés libres, ils n'auraient légalement pas dû avoir été asservis» (p. 240). Cette continuité entre Afrique et Amérique permet de résoudre l'apparent paradoxe de la faiblesse numérique des esclaves africains musulmans et le nombre relativement plus important de manumission qu'ils sont parvenus à obtenir avant même la mise en place de l'abolition.

15. Cette faiblesse numérique des esclaves musulmans fait dire à Paul Lovejoy que « L'»abolition» européenne a été possible parce que les gouvernements musulmans étaient opposés à la vente de quelque esclave que ce soit aux chrétiens, comme un moyen de protéger à la fois ceux d'entre les musulmans qui pourraient être mis en esclavage à tort et les esclaves qui auraient pu se convertir à I'Islam ultérieurement » (p. 238).

16. Cette dernière caractéristique étant développée plus récemment dans Lovejoy $P$. E., 2005, "The urban background of enslaved Muslims in the Americas », Slavery and Abolition, 26(3), p. 349-76. 
L'étude de Yacine Daddi Addoun et Paul E. Lovejoy sur un manuscrit arabe écrit autour de $1823^{17}$ par Muhammad Kabâ Saghanughu, esclave dans une plantation des environs de Kingston et leader de la communauté musulmane locale, permet de mieux saisir ce processus. Originaire d'une famille de clercs mandingues reliée à un courant quiétiste de la Tarîqa Qâdiriyya ${ }^{18}$, il arrive en Jamaïque comme esclave en 1777. En 1812, dans le contexte d'une dispute entre esclaves et propriétaires, il trouve assistance auprès d'une mission chrétienne morave, suite à quoi il se convertit au christianisme. Pour les auteurs, la trajectoire de Kabâ illustre le comportement des esclaves musulmans en Jamaïque qui « ont utilisé le langage parlé pour transmettre un message d'adaptation (accommodation) et d'adhésion à la chrétienté tout en utilisant l'arabe écrit et des symboles particuliers, tels que les noms, pour clamer leur autonomie religieuse et leur supériorité spirituelle en tant que musulmans » (p. 207). Kabâ s'institue alors comme un véritable intermédiaire entre les esclaves et les propriétaires: il fréquente les premiers dans les champs et les seconds à l'église, préférant, en raison de ces appartenances multiples, ne pas participer aux révoltes d'esclaves de 1831-32.

Dans cette optique, les comportements de résistance, ou de compromis avec les maîtres, ou encore les stratégies de retour en Afrique au moment de I'abolition s'expliqueraient par la modulation des systèmes d'attentes acquis en Afrique de l'Ouest et expérimentés aux Amériques. Les auteurs distinguent deux types de réactions des esclaves musulmans africains. La première, davantage expérimentée par les esclaves en provenance de terres du jihâd comme le Hausaland, comportait un fort engagement résistant allant jusqu'à la fomentation de révoltes (Bahia, 1835). La seconde, davantage exploitée par des esclaves provenant des traditions musulmanes plus quiétistes du pays Mandingue, consistait à rechercher, sur le modèle établi en Afrique de l'Ouest, des relations de compromis avec les maîtres évoluant sur le registre patron/ client. Selon Paul E. Lovejoy (dernier chapitre), I'opposition des deux stratégies n'est pas inéluctable et il n'est pas sûr que les stratégies d'ajustements n'aient pas aussi servi la recherche d'autonomie, d'émancipation et de retour en Afrique. Ce faisant, certains esclaves musulmans ont pu se faire les porteparole de leur communauté auprès de leurs maîtres afin de lui assurer un meilleur sort (comme l'assignation à des travaux qualifiés) tout en maintenant une identité musulmane que permettait d'entretenir l'utilisation de l'arabe, incompris des maîtres. C'est à la description de types d'ethos que tend le projet de Paul E. Lovejoy.

17. Le manuscrit porte le titre de Kitâb al-Salât (Le livre des prières).

18. Confrérie soufie d'Afrique de l'Ouest fondée par Abd al-Qâdir al-jîlânî (mort en 1166). 